\title{
Archives
}

\section{Acquérir, évaluer et conserver des photographies dans un milieu de santé et de services sociaux}

\author{
Nancy Gadoury
}

Volume 47, numéro 1, 2017

URI : https://id.erudit.org/iderudit/1041830ar

DOI : https://doi.org/10.7202/1041830ar

Aller au sommaire du numéro

Éditeur(s)

Association des archivistes du Québec (AAQ)

\section{ISSN}

0044-9423 (imprimé)

2369-9256 (numérique)

Découvrir la revue

\section{Citer cet article}

Gadoury, N. (2017). Acquérir, évaluer et conserver des photographies dans un milieu de santé et de services sociaux. Archives, 47(1), 153-183.

https://doi.org/10.7202/1041830ar
Résumé de l'article

Nancy Gadoury, bibliothécaire et archiviste au Centre intégré de santé et de services sociaux de Lanaudière, nous présente les étapes qui ont mené à la diffusion de photographies conservées au fil des années par les différentes entités composant le centre. À la suite d'une réorganisation administrative des établissements de santé et de services sociaux, un projet de gestion des documents administratifs a été mis en branle, projet qui comprenait, en plus de la révision des outils de gestion documentaire et du réaménagement des locaux, le traitement et la diffusion de la collection de photographies. L'auteure survole donc les moyens mis en oeuvre pour atteindre cet objectif, dans un contexte où les ressources sont limitées. La collection comprenant plus de 11000 photographies, dont certaines conservées dans des conditions mettant en danger leur conservation à long terme, le travail a été organisé en tenant compte du contexte particulier des milieux de santé. Ce contexte a notamment eu un impact sur les critères de tri ainsi que sur la façon de classer et de décrire les pièces. L'équipe en charge de ce projet a dû faire appel à de nombreux employés actuels et retraités afin de procéder à l'indexation des photographies de façon plus précise. Ce texte expose les réussites ainsi que les difficultés rencontrées dans ce processus et dresse un bilan somme toute positif de l'expérience compte tenu du potentiel évocateur des images et de la lumière différente qu'elles jettent sur les services de santé qui sont souvent perçus négativement par le public.
Ce document est protégé par la loi sur le droit d'auteur. L'utilisation des services d'Érudit (y compris la reproduction) est assujettie à sa politique d'utilisation que vous pouvez consulter en ligne.

https://apropos.erudit.org/fr/usagers/politique-dutilisation/ 


\section{NOTE ET BILAN D'EXPÉRIENCE}

\section{Acquérir, évaluer et conserver des photographies dans un milieu de santé et de services sociaux}

\section{NANCY GADOURY}

Bibliothécaire et archiviste, Centre intégré de santé et de services sociaux de Lanaudière.

\section{INTRODUCTION}

Découvrir une collection de photographies jamais traitées, lorsqu'un archiviste devient responsable d'un fonds d'archives, c'est un peu comme tomber sur un vieux trésor.

Les archivistes, comme leurs concitoyens d'ailleurs, éprouvent un attachement plus grand (qui frôle parfois l'irrationnel) envers les photographies qu'envers les autres supports d'information puisque des gens, des événements et des objets porteurs de "souvenirs» y sont montrés. (Charbonneau et Robert, 2003)

L'équipe de gestion des documents administratifs (GDA) du Centre de santé et de services sociaux (CSSS) du Nord de Lanaudière, qui a fait 
cette découverte, a tout de suite été enchantée. Un peu comme dans un cliché d'images d'archives, les photographies avaient été empilées dans des boîtes, entreposées dans un local oublié, conservées dans un vieux tiroir ou gardées par des employés nostalgiques. Malgré la charge de travail qu'elles représentaient, l'équipe a rapidement émis une procédure pour les récupérer.

Leur valeur était incontestable pour l'histoire de l'organisation et plus encore. Les photographies portaient les souvenirs de la mise en place du réseau de santé et de services sociaux pour toute la région nord de Lanaudière, un territoire de près de 12000 km carrés, regroupant quatre municipalités régionales de comté (MRC d'Autray, de Joliette, de Matawinie et de Montcalm) incluant plus de 50 municipalités et une communauté autochtone. Les plus anciennes photographies dataient même du début du XXe siècle, racontant l'implantation du premier hôpital installé à Joliette et géré par les Sœurs de la Providence, en passant par l'établissement de l'Hôpital Saint-Charles en 1959, événement marquant dans le cadre des soins psychiatriques et de la recherche en santé mentale au Québec.

Après les années 1980 et même après la fusion 2003-2004, les photographies étaient encore plus nombreuses. Ce qui était assez surprenant, c'est que ces photographies illustraient un tout autre côté de ce que nous avons l'habitude de voir du milieu de la santé. En tant que patients ou visiteurs, l'expérience que nous avons des hôpitaux, des centres locaux de services communautaires (CLSC) et des centres $d$ 'hébergement et de soins de longue durée (CHSLD) ne fait que très rarement partie de nos bons souvenirs. Ils sont souvent synonymes de maladies physiques ou mentales, de souffrances et d'inquiétudes pour nos proches malades. Les images véhiculées par les médias sur les milieux de santé et de services sociaux ne sont pas très positives non plus. Le milieu éprouve depuis plusieurs années diverses difficultés budgétaires, organisationnelles et administratives.

Pourtant, les photographies du fonds d'archives du CSSS du Nord de Lanaudière racontent une tout autre histoire: celle des gens qui se dévouent chaque jour pour soigner les autres, celle des gens qui construisent de nouveaux édifices pour mieux accueillir les gens malades, celle des gens qui développent de nouveaux services, celle des médecins qui introduisent des traitements et des méthodes de soins plus efficaces, 
celle de bienfaiteurs qui, grâce à leur générosité, permettent l'achat d'équipements de pointe, et celle d'une équipe énorme qui travaille avec cœur malgré les coupures de budget et de personnel qui perdurent depuis des années.

Devant une telle découverte, I'archiviste n'a qu'un seul choix: dévoiler cette collection d'images inédites pour rétablir l'équilibre. Après tout, des milliers de vies commencent dans les hôpitaux et des milliers de gens en sortent guéris.

Toutefois, rassembler, traiter, protéger cette collection et la rendre disponible n'était pas une mince affaire. La situation du Service de gestion des documents administratifs du CSSS du Nord de Lanaudière ne permettait pas d'envisager un projet de traitement et de diffusion de cette collection rapidement et facilement. Au moment de cette découverte, seulement un archiviste et un technicien en documentation, tous deux à temps partiel, étaient responsables de la gestion des documents administratifs pour toute l'organisation, qui comptait 5000 employés dispersés dans 26 établissements. II n'y avait aucun budget d'acquisition attribué au service et l'équipe arrivait difficilement à répondre aux demandes courantes. Par ailleurs, le milieu de la santé et des services sociaux investit un faible taux de son budget dans les projets administratifs (non cliniques ou n'ayant aucun lien avec les soins aux patients). Bref, le contexte n'était pas très favorable.

Pourtant, après de nombreux efforts, la collection a pu être entièrement traitée et est maintenant disponible à la consultation. Dans cet article, les différentes étapes qui ont mené à la réalisation de ce projet de traitement de la collection de photographies du CSSS du Nord de Lanaudière seront présentées. Tout d'abord, le projet de gestion des documents administratifs déployé au CSSS du Nord de Lanaudière sera décrit, car le projet de traitement des photographies en faisait partie et a bénéficié des avancements de ce projet de plus grande envergure. Les principes d'acquisition des photographies ayant été mis en place, les méthodes de classification et de description seront également décrites. Une attention particulière a été portée aux critères d'évaluation utilisés pour le tri des photographies, parce que c'était un enjeu majeur de notre projet et étant donné le temps de travail consacré à cette étape. Puis, quelques détails sur les moyens de conservation choisis et de diffusion seront donnés. 


\section{PROJET DE GESTION DES DOCUMENTS ADMINISTRATIFS DU CSSS DU NORD DE LANAUDIÈRE}

\subsection{Historique}

Comme plusieurs organismes publics, le premier projet de gestion des documents administratifs qui a été mis en place dans le nord de Lanaudière date de 1995, avec l'entrée en vigueur de la Loi sur les Archives (RLRQ, chapitre A-21.1) telle qu'elle est appliquée aujourd'hui. À cette époque, un plan de classification et un calendrier de conservation avaient été faits par la firme Gestar pour répondre aux besoins du centre hospitalier régional de Lanaudière (CHRDL), I'unique hôpital du nord de Lanaudière. Au même moment, le logiciel de gestion documentaire Coba avait été acquis. Ce premier système de gestion documentaire a été déployé dans quelques directions du CHRDL entre 1995 et 2003.

Cependant, à la suite du départ du gestionnaire de projet et des nouvelles fusions créant les centres de santé et de services sociaux en 2003-2004, ce premier projet de gestion des documents administratifs est tombé quelque peu dans l'oubli. En bref, ceux qui avaient été formés au système crée par Gestar au CHRDL continuèrent à I'utiliser, mais aucun autre employé ne fut formé et le déploiement fut abandonné. Ce fut le même constat dans les autres établissements fusionnés en 2003-2004 (CLSC et CHSLD). Les systèmes qui y avaient été mis en place continuèrent à être utilisés marginalement en parallèle à celui du CHRDL.

Le mandat de la gestion des documents administratifs finit par être mis entre les mains de la bibliothèque du CHRDL vers 2006. Toutefois, aucune ressource ne fut attribuée au projet. Donc, entre 2003 et 2009, seul un technicien en documentation à temps partiel continua de s'assurer du bon fonctionnement du système de gestion des documents administratifs. Son principal mandat était de faciliter l'entrée des boîtes de documents à la voûte des semi-actifs, ainsi que de détruire les documents une fois le délai de conservation échu. Aucun projet de mise à jour et de fusion des systèmes de gestion des documents administratifs ne put être envisagé.

Évidemment, plusieurs problématiques non réglées au cours de ces années devinrent bientôt trop importantes pour les ignorer: nombreux systèmes de gestion des documents administratifs entrainant la confusion chez les employés, impossibilité d'obtenir de la formation et du soutien 
pour ces systèmes, plans de classification et calendriers de conservation multiples et non adaptés à la nouvelle réalité des CSSS, désuétude des logiciels utilisés, problèmes récurrents de repérage des documents, difficulté d'identification des documents à détruire et à conserver, débordement des locaux d'entreposage, entreposage non sécuritaire, destruction aléatoire des documents, problèmes de confidentialité, etc.

Les nombreuses tensions et insatisfactions des utilisateurs des systèmes de gestion des documents administratifs finirent par convaincre la direction d'embaucher un archiviste pour remédier à la situation en 2009. Malheureusement, aucun des outils des systèmes de gestion des documents administratifs (plan de classification, calendrier de conservation et logiciel) existant dans l'organisation n'était adéquat pour remédier aux diverses problématiques. II a donc fallu recommencer depuis le début et présenter un tout nouveau projet de gestion des documents administratifs.

\section{2. Élaboration du projet de gestion des documents administratifs}

Le défi initial de ce projet fut de convaincre la direction du CSSS du Nord de Lanaudière qu'il ne suffisait pas de faire le ménage des locaux pour régler la situation, mais bien de mettre en place un réel système de gestion des documents administratifs, soutenu par une équipe adéquate. En se basant sur les articles de la Loi sur les archives (RLRQ, chapitre A-21.1), la Loi sur l'accès aux documents des organismes publics et la protection des renseignements personnels (RLRQ, chapitre A-2.1), ainsi que sur la Loi concernant le cadre juridique des technologies de I'information (RLRQ, chapitre C-1.1), et les obligations que doivent remplir les organismes de santé et de services sociaux en ce qui concerne la gestion de leurs documents administratifs, il a été possible de les convaincre de commencer une refonte des systèmes existants.

Le projet présenté comportait treize étapes:

1. Mise à jour du plan de classification

2. Mise à jour du calendrier de conservation

3. Réaménagement des locaux d'entreposage d'archives

4. Acquisition d'un nouveau logiciel 
5. Révision de la politique de l'information administrative

6. Gestion des risques: élaboration d'un plan de mesures d'urgence pour les documents administratifs

7. Attribution d'un budget et des ressources humaines au service

8. Implantation du système de gestion des documents administratifs

9. Création d'un fonds d'archives historiques et tri des documents non traités

10. Traitement de la collection de photographies

11. Mise en place du suivi du système de GDA, déclassement, versement annuel et mise à jour des outils

12. Mise en valeur du système de gestion des documents numériques

13. Mise en valeur des archives historiques

Plusieurs présentations au comité de direction du CSSS du Nord de Lanaudière ont été nécessaires pour faire le tour du projet et plusieurs documents de projets différents ont été rédigés pour répondre aux critères de sélection de chacune des directions engagées dans le projet (par ex. : projet de réaménagement des locaux présenté à la Direction des services techniques, projet d'acquisition d'un nouveau logiciel à la Direction des ressources informationnelles, etc.).

\section{3. Étapes 1 et 2 : plan de classification et calendrier de conservation}

Franchir les deux premières étapes du projet a été difficile et très long. Seulement deux jours de travail par semaine ont été attribués à l'archiviste pour le projet, le technicien en documentation continuant ses tâches habituelles. II a fallu dix-huit mois pour compléter la mise à jour du plan de classification et la mise à jour du calendrier de conservation.

Le milieu de la santé et des services sociaux est un milieu très politisé et règlementé dans lequel il est souvent difficile de pénétrer. La plupart des services et directions fonctionnent en silo, rendant laborieuses la collaboration ou la mise en commun des efforts et la productivité de ceux-ci. Chacun des sous-services possède un ou des systèmes propres (logiciels, numérotation des dossiers, lois et exigences de conformité, etc.) 
avec lesquels l'archiviste doit composer. Bref, pour chaque service, et parfois même sous-service, il a fallu étudier les processus de gestion documentaire afin de bien adapter les outils proposés.

Les gestionnaires sont également surchargés, donc très peu disponibles, autant pour exprimer leurs problématiques en gestion des documents administratifs que pour entendre les propositions d'amélioration. Comme très peu d'accent est mis sur les projets de nature administrative, il a été ardu, même pour les gestionnaires intéressés, de faire de la place pour un projet de gestion des documents administratifs, malgré les nombreux avantages qu'il comportait pour chacun. II faut dire aussi que la taille de l'organisation (rappelons-le, 26 établissements fusionnés et plus de 5000 employés) a rendu la planification du projet plutôt ardue. Même en 2009, plus de cinq ans après la fusion, plusieurs directions et services du CSSS du Nord de Lanaudière n'avaient toujours pas complété la refonte de leurs activités, compliquant encore plus les processus de mise à jour du système de gestion des documents administratifs. Difficile de faire une proposition de gestion documentaire unifiée, quand les services fonctionnent encore eux-mêmes avec plusieurs méthodes différentes.

Pour la mise à jour du plan de classification et du calendrier de conservation, il a donc fallu autant se fier aux outils existants (plans et calendriers des établissements fusionnés), qu'au Recueil des règles de conservation des documents des établissements de santé et de services sociaux du Québec (AQESSS, 2009), qu'aux différentes incursions faites dans chacun des services. La partie du plan de classification et celle des délais de conservation liées à nos activités particulières (santé et services sociaux) ont été développées en grande majorité originellement à partir de ces derniers entretiens, rallongeant ainsi le processus de mise à jour de ces outils. L'approbation du plan de classification et du calendrier de conservation a pu être effectuée au début de l'année 2011 auprès de Bibliothèque et Archives nationales du Québec. À partir de ce moment-là, il a été possible de gérer plus efficacement (et légalement) l'entrée et la sortie des documents des locaux d'entreposage et d'éviter leur surcharge. 


\section{4. Étape 3: réaménagement des locaux d'entreposage d'archives}

L'autre priorité du projet de gestion des documents administratifs était le réaménagement de nos locaux d'entreposage surchargés et inadéquatement équipés. Tout d'abord, les deux locaux sous la responsabilité du Service de gestion des documents administratifs ne respectaient pas les normes d'entreposage minimales pour les documents d'archives (Archives nationales du Québec, 2000). Un des entrepôts était situé dans un immeuble désaffecté et l'autre était équipé d'étagères non conformes. Aucun des deux n'avait de protection (gicleurs) contre les incendies.

De plus, l'espace d'entreposage disponible ne correspondait pas aux besoins réels de l'organisation (qui étaient près du double). Plusieurs boîtes et documents administratifs étaient éparpillés dans les différents établissements, dans les bureaux du personnel et même dans les couloirs, sans protection et sans sécurité adéquate.

Nous avons dû remédier rapidement à la situation et proposer des projets de réaménagement pour les locaux. Nous avons réorganisé complètement un des locaux à notre disposition avec des étagères et des dispositifs de protection contre les incendies avec l'aide de la Direction des services techniques en 2010. Un second espace nous a également été attribué en 2011 et a été équipé de la même façon.

Les boîtes entreposées dans l'immeuble désaffecté, ainsi que celles éparpillées dans les établissements, les bureaux et les couloirs ont été rapatriées dans nos locaux au cours des mois suivants. Au total, avec ces réaménagements, nous avons pu doubler la capacité d'entreposage pour le même nombre de pieds carrés occupés. Ces espaces réaménagés nous ont également permis de pouvoir rapatrier la totalité de la collection de photographies sous notre responsabilité et d'en faire un inventaire sommaire.

\section{5. Étape 4 : acquisition d'un nouveau logiciel}

Dès les premiers mois du projet de gestion des documents administratifs, un projet d'acquisition d'un nouveau logiciel a été présenté à la Direction des ressources informationnelles (DRI). La plupart des projets informatiques 
déposés à la DRI prennent plusieurs mois à être évalués, étudiés, financés et mis en place. Notre projet d'acquisition a été accepté en 2011, soit près de deux ans après sa présentation. Coba et les autres logiciels de gestion documentaire présents dans les différents établissements ont finalement été remplacés progressivement par IntelliGID de Doculibre, un logiciel libre. Ce critère a été déterminant dans le choix du logiciel étant donné qu'aucun budget n'était disponible pour ce genre d'achat.

Quoi qu'il en soit, IntelliGID a répondu adéquatement à nos besoins de gestion de documents administratifs. Le logiciel a été installé à peu près au même moment où nous avons commencé le traitement de la collection des photographies. Donc, plusieurs des étapes du projet de traitement de la collection ont pu être développées en fonction des possibilités que nous offraient ce nouvel outil.

\section{6. Étapes 5 et 6 : politique de gestion des documents administratifs et plan de mesures d'urgence}

Une politique de l'information administrative existait depuis 1995 au CHRDL. Elle a été révisée et finalement approuvée par le comité de direction du CSSS du Nord de Lanaudière en 2011. Remettre la politique à jour nous a permis de nous adjoindre plusieurs directeurs dans la poursuite de notre projet de gestion des documents administratifs, car une de leurs responsabilités est de faire appliquer les différentes politiques et procédures dans leur direction. Une fois la politique approuvée par le comité de direction, les directeurs pouvaient plus difficilement se soustraire à l'exercice que nous leur proposions.

À partir de cette politique, plusieurs procédures ont été rédigées pour uniformiser les processus de gestion des documents administratifs dans tous les établissements fusionnés: le plan de mesures d'urgence (Casault et Charbonneau, 2001), la procédure de versement des documents au semi-actif et à l'historique, ainsi que la procédure de traitement des photographies et des archives audiovisuelles.

\section{7. Étapes 7 et 8 : ressources et implantation}

Malheureusement, encore aujourd'hui, les ressources octroyées au projet de gestion des documents administratifs sont très minces. Malgré 
ces contraintes, l'implantation du système de gestion des documents administratifs, ainsi que la formation aux utilisateurs, ont tout de même pu débuter à la fin de l'année 2012, mais la progression a été très lente. Avec cinq jours de travail (deux pour l'archiviste et trois pour le technicien), il a été possible de former seulement entre dix et quinze personnes par année, ce qui était équivalent à un sous-service par trimestre, à une direction par an, sur un total de treize directions.

Le temps de formation n'était en aucun cas problématique (3,5 heures par groupe de 5 personnes). Ce fut plutôt le soutien après la formation aux divers utilisateurs qui a causé des débordements de travail pour l'équipe. Entre 2012 et 2014, les demandes de soutien ont augmenté de $300 \%$, réduisant considérablement le temps consacré à faire les approches aux services et aux directions à former. Les versements de documents ont aussi augmenté pour la même période de $65 \%$ environ.

Étant donné que le système de gestion des documents administratifs avait périclité durant de nombreuses années, il y avait énormément de retard à rattraper dans chacun des services formés. Bref, à chaque fois que des utilisateurs recevaient une formation, ils en profitaient pour faire un grand ménage et revoir tous leurs processus de gestion documentaire. Le soutien offert par l'équipe de gestion des documents administratifs était alors grandement sollicité pendant plusieurs semaines. Une quantité énorme de documents était également versée, sous tous les formats (dont des photographies), datant même de plusieurs décennies (là où le premier projet de gestion documentaire ne s'était jamais rendu). Le retard accumulé dans le traitement des documents semi-actifs et historiques a été énorme dès les premiers mois de l'implantation du projet.

Néanmoins, I'amélioration de la gestion des documents administratifs a été notoire pour tous les services où le système a été implanté: fin des épisodes de destruction aléatoire des documents, diminution radicale de la perte de temps pour le repérage des documents, grande satisfaction des utilisateurs face au nouveau système, classement uniforme des documents, fin de l'encombrement de nos locaux et des espaces de classement des services (application facile du calendrier), amélioration des processus de transfert des documents dans les locaux d'entreposage, élimination des situations dangereuses en lien avec la confidentialité, etc. 


\section{8. Étapes 9 et 10: création d'un fonds d'archives historiques et de la collection de photographies}

Dès qu'il a été possible de commencer à traiter les archives historiques, avec I'installation d'IntelliGID principalement, le fonds du CSSS du Nord de Lanaudière a été créé. Tout comme pour les photographies, beaucoup de documents papier (plus de 1500 boîtes) avaient été accumulés dans les différents établissements et avaient été envoyés au Service de gestion des documents administratifs lors du rapatriement des archives dans nos nouveaux locaux réaménagés. Nous avons débuté le traitement des documents historiques papier les plus récents pour tester nos outils, ce qui a contribué à l'amélioration de ceux-ci avant l'implantation réelle.

Toutefois, plusieurs autres fonds restent à créer et à traiter, car nous possédons maintenant tous les documents des établissements fusionnés en 2003-2004. Malheureusement, les fonds des établissements fusionnés ont été mélangés avec les années, et aucun n'a été traité. Même au CHRDL, le contenu des boîtes n'est pas uniforme et contient des documents provenant des hôpitaux précédents: I'Hôpital Saint-Charles et l'Hôpital Saint-Eusèbe. Seule la distinction avec l'année des documents nous permet désormais de savoir à quel fonds ils appartiennent. Encore aujourd'hui, des centaines de boîtes n'ont jamais été ouvertes et leur contenu demeure toujours inconnu.

Ce fut le même constat pour les photographies. Il était évident qu'elles provenaient de plusieurs fonds différents, mais comme elles arrivaient souvent pêle-mêle et de façon aléatoire au Service de gestion des documents administratifs, il a été décidé de créer une collection.

On affirme que les documents iconographiques (gravures, dessins, photographies, etc.) doivent être conservés par les services d'archives lorsqu'ils appartiennent à des fonds ou s'ils constituent par eux-mêmes des fonds, mais on reconnaît du même souffle le droit de constituer des collections iconographiques à propos de personnalités ou d'événements politiques. (Smith, 2008-2009)

La création d'une collection est appropriée étant donné que c'est le même sujet qui est représenté par toutes ces photographies: la santé et les services sociaux dans Lanaudière. 


\section{PROJET: ARCHIVES PHOTOGRAPHIQUES}

Parallèlement à la rédaction du projet de gestion des documents administratifs, le projet de traitement des archives photographiques a été élaboré. Pourquoi centrer l'attention si rapidement sur le traitement de la collection de photographies, alors que beaucoup d'autres enjeux entraient en ligne de compte?

Premièrement, l'urgence de la situation a poussé l'équipe à agir plus rapidement. Entre 2009 et 2012, il a été possible, pour le Service de gestion des documents administratifs, de récupérer plus de 11000 photographies sur différents supports. Environ $75 \%$ de cette collection se trouvait dans un local ayant été sous la responsabilité du technicien en audiovisuel parti à la retraite cinq ans plus tôt. Avant de vider le local (et de jeter ce qui s'y trouvait), la Direction des services techniques nous a accordé quelques semaines pour nous permettre d'identifier ce qui était à conserver. Les photographies retenues ont dû être entreposées dans les locaux de la bibliothèque, car les locaux d'archives n'étaient toujours pas réaménagés. L'encombrement était de taille.

De plus, la plupart des photographies récupérées étaient entreposées dans les enveloppes papier de la compagnie qui faisait le développement des épreuves, ce qui était loin des conditions idéales pour la conservation. Parfois, elles étaient dans des boîtes, des pots de plastiques, des cartables, des albums et même des cadres. II y avait des photographies de tous les formats: négatifs, diapositives, sur disquettes, sur CD, etc. Plusieurs signes de détérioration étaient déjà visibles pour plusieurs d'entre elles.

Les employés de l'organisation venaient également nous questionner fréquemment sur le contenu des boîtes, demandaient d'y jeter un œil, de consulter et de reproduire des photographies. Traiter et rendre disponible ces photographies est rapidement devenu une clé d'entrée, un moyen d'introduction à notre projet de gestion des documents administratifs. "L'utilisation des archives photographiques, si elle est bien vendue aux décideurs de l'organisme parrain du service d'archives, permet à celui-ci de gagner en notoriété auprès des décideurs » (Charbonneau et Robert, 2003). Dès que les premiers lots furent traités, ils ont servi à démontrer à plusieurs gestionnaires et services ce que permettait l'investissement de temps dans notre projet. 


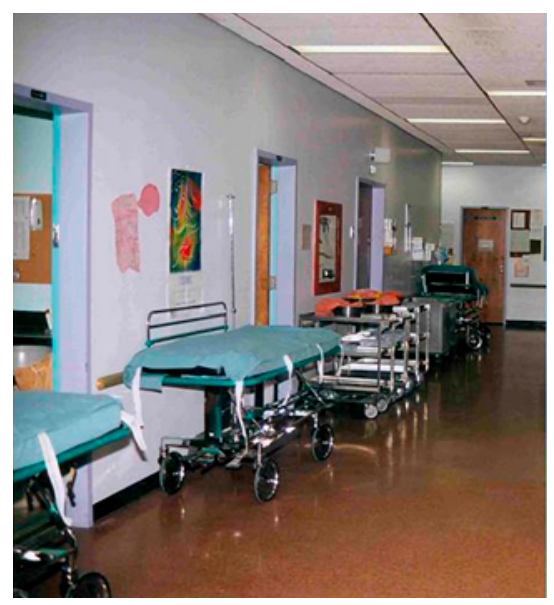

Figure 1: Local du Bloc opératoire au CHRDL, mai 2000

Les images qui fixent I'histoire d'un service et insistent sur le souvenir, sur les réalisations de chacune des personnes travaillant dans l'organisation, sont beaucoup plus séduisantes qu'une succession de boîtes de documents papier bien classées sur des tablettes.

\subsection{Acquisition des photographies}

Au départ, le Service de gestion des documents administratifs n'a pas été très sélectif à l'acquisition des archives photographiques. L'équipe a plutôt opté pour des critères d'acquisition très larges. Le but était d'avoir une vue d'ensemble de la collection pour définir par la suite quelles photographies reflétaient le mieux la réalité de l'organisation, les activités de soins et services sociaux, et quelle était l'utilisation que le service pouvait en faire.

Dans un premier temps, la récupération d'urgence des documents dans le local du technicien en audiovisuel a permis de récupérer les photographies des événements marquants ayant ponctué l'évolution de I'Hôpital Saint-Charles et du CHRDL des années 1970 aux années 2000.

II y avait également plusieurs photographies illustrant le personnel médical en action, les divers locaux à travers le temps, les services offerts aux patients, autant que des photographies plus techniques illustrant, par exemple, les repas offerts à la cafétéria. 
Tous les moyens ont été utilisés pour récupérer le plus grand nombre de photographies possibles: messages aux employés (par ex. : notes de service, bouche à oreille et visite des gestionnaires durant le projet de gestion des documents administratifs), promenade dans les couloirs et les bureaux pour ramasser les cadres «désuets », exploration des dossiers du service des communications (photographies de couvertures des événements, publicités et publications), approches auprès des ressources humaines qui gèrent les événements de reconnaissance et les formations (photographies des fêtes des retraités, remise de prix d'excellence et formations offertes), création de liens avec le club social (photographies d'activités sociales et des loisirs offerts aux employés), incursion dans les méthodes de travail de la Direction des services techniques (photographies des projets de construction et de rénovation), visite à la Direction générale (portraits officiels des directeurs et comités et albums photos historiques), ainsi que la création de contacts avec les équipes médicales (photographies des soins offerts et formations). Toutes les directions et tous les sous-services se sont prêtés à l'exercice avec enthousiasme. La plupart des employés n'avaient pas jeté les photographies, y attachant une valeur sentimentale, et étaient heureux de savoir qu'elles bénéficieraient d'un traitement privilégié.

Le seul frein rencontré par l'équipe pour l'acquisition des photographies a été du côté médical. Plusieurs photographies illustrant des patients ont fait office de discussions, étant donné le principe de confidentialité des soins. II s'agissait surtout de photographies prises dans le cadre d'un soin pour documenter le dossier du patient ou pour permettre l'identification du patient, ou encore pour une procédure légale. Après mûre réflexion, il a été décidé de détruire les photographies existantes sur lesquelles I'identification des patients était possible, ainsi que les photographies prises pour documenter le dossier d'un patient, et elles ont été exclues des acquisitions.

Les photographies illustrant des chirurgies ou des soins de base ont aussi été rejetées et ne font plus partie des acquisitions du Service de gestion des documents administratifs. D'abord, elles sont vraiment difficiles à repérer; elles ne font pas partie d'un processus habituel de prestation des soins. Elles sont prises aléatoirement par différents employés et médecins selon des besoins ponctuels. Ensuite, dans la majorité des cas, il n'y a aucun élément de description qui les accompagne. II faut souvent une formation médicale pour en comprendre le sens. Finalement, l'équipe a aussi constaté qu'il y avait très peu d'intérêt des utilisateurs pour ce type de photographies. 


\subsection{Classification des photographies}

Comme la collection de photographies a été récupérée çà et là dans divers services du CSSS du Nord de Lanaudière, et qu'il n'y avait pas de regroupements préétablis, l'équipe a choisi d'utiliser le nouveau plan de classification de l'organisation pour y mettre de l'ordre (Charbonneau et Robert, 2003). Comme le plan de classification du CSSS du Nord de Lanaudière rassemblait toutes les activités de santé et de services sociaux des anciens établissements fusionnés, il était logique de croire qu'il serait possible d'y classer toutes les photographies acquises, quelle que soit leur provenance.

Les cotes de classification ne suffirent toutefois pas pour distinguer clairement les différentes séries de photographies. Par exemple, la cote G7 302, qui correspond aux projets de construction, contenait plusieurs séries avec des titres très semblables: réaménagement du sous-sol, changement de la fenestration, travaux de peinture, etc. II a donc été décidé d'attribuer un numéro séquentiel à chacune des séries dans le but de les distinguer.

Le même constat a été fait au niveau de la pièce. Certaines séries contenaient des dizaines et parfois même des centaines de photographies. Pour les différencier plus aisément, un numéro séquentiel a été attribué à chacune des photographies. La numérotation des pièces a aussi facilité grandement la description à la pièce et le repérage ensuite.

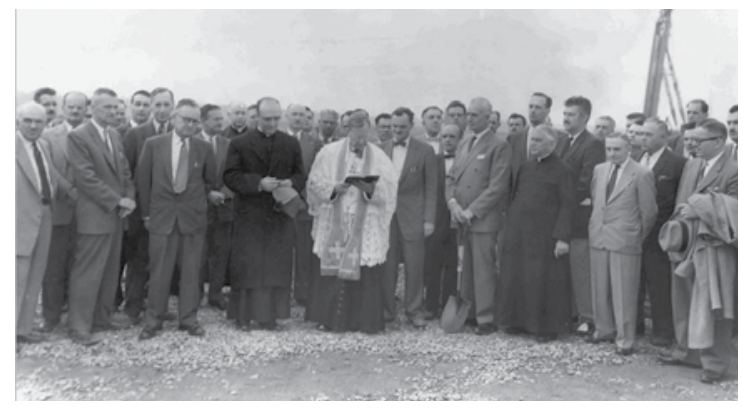

Figure 2: Bénédiction de la pierre angulaire du futur Hôpital Saint-Charles par Mgr Joseph-Arthur Papineau, 23 octobre 1959

Par exemple, la photographie ci-dessus a pour cote complète le G3 300 - S02 - P03. Dans le plan de classification du CSSS du Nord 
de Lanaudière, la série a été classée sous la cote G3 300, qui correspond aux cérémonies et événements officiels. II s'agissait de la deuxième série que l'équipe traitait qui avait cette cote, d'où le S02 (série $n^{\circ} 2$ ). La photographie est la troisième photo de la série, d'où le P03 (photographie $n^{\circ} 3$ ).

\subsection{Description et indexation des photographies}

Pour la description des photographies, I'équipe a utilisé les Règles pour la description des documents d'archives (RDDA). Lors de la rédaction du projet, comme IntelliGID n'était pas encore disponible, l'équipe a créé une fiche de description à partir de Word avec les champs choisis. Ces fiches ont ensuite été insérées dans IntelliGID qui permet la recherche plein texte dans les documents numériques.

Voici un exemple de fiche remplie, ainsi qu'une des photographies de la série décrite.

\section{Collection du CSSS du Nord de Lanaudière}

\begin{tabular}{|l|l|}
\hline Cote: & P1 304 - S01 \\
\hline $\begin{array}{l}\text { Titre de } \\
\text { la série: }\end{array}$ & $\begin{array}{l}\text { Activités sociales pour les patients du service de soins de longue durée en psychiatrie de } \\
\text { l'Hôpital Saint-Charles }\end{array}$ \\
\hline Photographe: & [après 1959] \\
\hline Date de création: & 13 photographies : épreuves n\&b; 21,5 X 25,5 cm \\
\hline Collation: & $\begin{array}{l}\text { Cette série comprend } 13 \text { photographies du service de soins longs durés en psychiatrie de } \\
\text { I'Hôpital Saint-Charles à Saint-Charles-Borromée. Les photos représentent des activités } \\
\text { sociales que les infirmiers et les infirmières ont organisées pour les patients du service de soins } \\
\text { de longue durée en psychiatrie. Les activités se sont déroulées entre autres à la cafétéria et au } \\
\text { gymnase de l'hôpital. }\end{array}$ \\
\hline Historique:
\end{tabular}

Identification des personnes ou des lieux :

\begin{tabular}{|l|l|}
\hline P01: & Cafétéria où se déroule une activité \\
\hline P02: & Volley-ball au gymnase \\
\hline P03: & Peinture \\
\hline P04: & Four à poterie \\
\hline P05: & Comité des bénéficiaires \\
\hline P06: & Bénéficiaire qui fait de la peinture \\
\hline P07: & Bowling au rez-de-chaussée C-01 \\
\hline P08: & \\
\hline P09: & \\
\hline P10: & Métier à tisser \\
\hline P11: & Coin livre pour les bénéficiaires \\
\hline P12: & Service de coiffure pour les bénéficiaires \\
\hline P13: & Salle des bénéficiaires et leur famille \\
\hline
\end{tabular}

Figure 3: Fiche de description des séries 


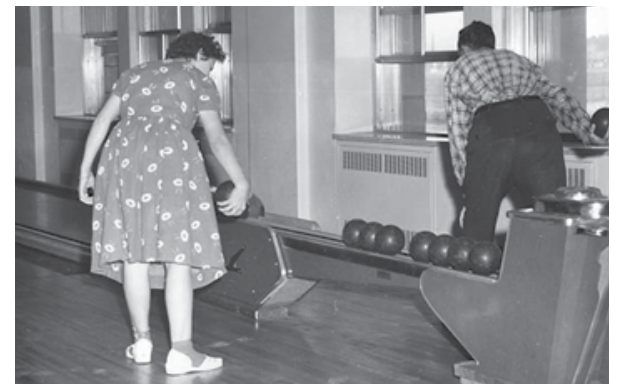

Figure 4: P1 304 - S01 - P07 Bowling au rez-de-chaussée C-01, [après 1959]

Les champs utilisés sont le titre de la série, le nom du créateur (photographe), la date de création, la collation et l'historique. Les quatre premiers champs ont été choisis, dans un souci de normalisation, pour identifier et distinguer chacune des séries et en connaître leur étendue. Le champ " historique » a été retenu pour illustrer le contexte de réalisation des photographies. Le but était d'inscrire chaque série « dans la mémoire organique et consignée de l'organisme, en documentant le contexte de sa création » (Smith 2008-2009).

La plupart du temps, les éléments de description ont été facilement repérables. Par exemple, pour les photographies provenant du local du technicien en audiovisuel, plusieurs notes manuscrites avaient été laissées sur les enveloppes: titre, date, personne ou service à qui les photographies étaient destinées. Les photographes ont aussi été facilement identifiables, étant donné que le même technicien en audiovisuel a été à l'emploi de I'hôpital pendant de longues années. Les autres photographies étaient, dans plusieurs cas, réalisées par des photographes professionnels dont les inscriptions étaient bien visibles.

Pour l'indexation, l'équipe s'est principalement basée sur les besoins des utilisateurs. Bien avant que la collection ne soit disponible à la consultation, plusieurs utilisateurs demandaient déjà de la voir. Trois principales demandes revenaient fréquemment: des photos des grands événements, des personnes et des emplacements. En bref, les utilisateurs voulaient soit commémorer un événement important ayant eu lieu dans leur service, soit avoir des photographies d'eux-mêmes ou de leurs collègues, soit voir l'évolution des bâtiments et des locaux au cours des années.

Pour répondre à ces demandes, il a été évident dès le départ que l'équipe devait faire une indexation au niveau de la pièce par sujet 
(Schwartz, 2002). Les titres choisis pour les séries, ainsi que l'historique, ne répondaient que partiellement à ces besoins, surtout en ce qui a trait à l'identification des personnes. Plusieurs séries étaient également hétérogènes comme celle dont la fiche de description est donnée en exemple ci-dessus (Figure 1). Plusieurs lieux et équipements différents y sont illustrés, même s'ils sont tous liés aux activités sociales offertes aux patients. Sans la description à la pièce par sujet, plusieurs photographies n'auraient pas été repérables.

La plupart des séries liées à la santé et services sociaux étaient aussi hétérogènes. Par exemple, deux séries ayant un titre semblable, tel que "Service de chirurgie», pouvaient contenir des photographies très différentes, des illustrations des locaux dans le premier cas et de l'équipe de chirurgie dans le deuxième cas.

Sans l'indexation à la pièce, les lieux et les personnes présentes sur les photographies auraient été difficiles à repérer. II existe une série de photographies pour le Gala Méritas chaque année sur presque vingt ans. Comme il y a en moyenne entre vingt et soixante photographies par série (après le tri), il aurait fallu consulter des centaines de photographies pour trouver une personne en particulier.

Comme pour les éléments de description, il a été aisé pour l'équipe de définir les éléments d'indexation. D'abord, l'équipe a utilisé un vocabulaire libre, principalement parce que le moteur de recherche d'IntelliGID est très efficace pour faire le repérage par mots-clés, simplifiant l'indexation par sujet. Aussi, l'équipe était déjà capable, par elle-même, d'identifier plusieurs lieux, étant donné que plusieurs images illustraient notre propre lieu de travail. Plusieurs personnes sur les photographies, nos collègues, étaient aussi facilement identifiables.

Pour les photographies plus anciennes, ou pour celles que l'équipe n'arrivait pas à identifier, diverses stratégies de participation des employés du CSSS du Nord de Lanaudière ont été utilisées. Premièrement, une fiche d'identification des photographies a été développée. Dans cette fiche, l'équipe a repris les champs de description et d'indexation souhaités (mais avec des termes vulgarisés). Cette fiche était envoyée par courriel ou par courrier interne à un employé qui avait été identifié sur la photographie. L'employé devait (sous une base volontaire) nous remplir la fiche au meilleure de ses connaissances et nous la renvoyer. Le taux de réponse 
a été très bon et plusieurs des employés sollicités sont même venus voir les photographies traitées pour participer davantage à l'indexation de la collection. L'équipe a également sollicité l'aide des employés cumulant plusieurs années d'ancienneté, ainsi que des retraités.

Plusieurs éléments de description et d'indexation ont aussi été pris dans les journaux organisationnels, plusieurs événements couverts par le service des communications et le technicien en audiovisuel ayant eu droit à une mention dans ceux-ci. Certains documents historiques commémoratifs de l'organisation ont aussi été utilisés. Les photographies liées à une formation, comme celle ci-dessous, étaient aussi souvent accompagnées d'un cahier de formation ou d'une cassette sonore qui permettait à l'équipe de compléter de façon très détaillée la fiche d'identification de la série.

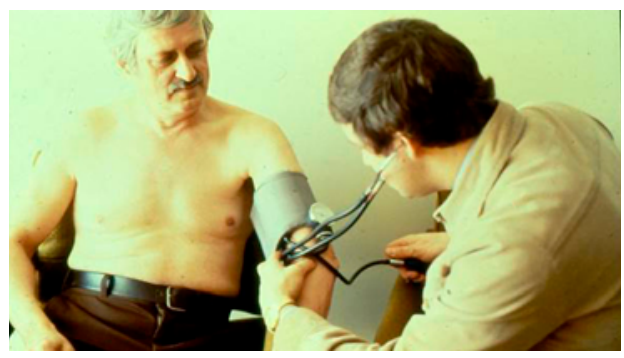

Figure 5: P1 905 - S01 - P29 Diapositive tirée de la formation Mieux vivre et convalescence à la maison suite à un infarctus, vers 1970

Ironiquement, les seules photographies que l'équipe a eu davantage de difficulté à décrire et indexer sont celles qui étaient plus récentes, donc en format numérique.

[...] avec I'arrivée du numérique, I'identification des photographies est de moins en moins systématique. On stocke le tout dans l'ordinateur sans prendre le temps de sélectionner, identifier, dater ou commenter. Au mieux, les photographies sont numérotées et datées lors de leur transfert de l'appareil à l'ordinateur. (Gadoury, 2009-2010)

La plupart des ensembles de photographies numériques ne dérogeait pas à cette règle. Seuls les employés contemporains à ces photographies (ou à l'événement couvert) étaient en mesure de faire une description sommaire à l'équipe. 


\section{4. Évaluation des photographies}

Cette étape du traitement des photographies a retenu un peu plus longuement l'attention de l'équipe du Service de gestion des documents administratifs lors de l'élaboration du projet. Avec 11000 photographies à traiter avec des ressources très limitées, cette étape devait être bien définie pour accélérer le plus possible le processus. En bref, le tri devait se faire rapidement et efficacement (pour libérer les espaces encombrés et limiter les dépenses en ressources humaines), et surtout être facilement réalisable par une personne provenant de l'externe (les ressources humaines à l'interne n'étant pas disponibles pour le faire).

L'équipe a donc élaboré un tableau à partir des critères de tri présentés par Mario Robert et Normand Charbonneau dans La gestion des archives photographiques (Charbonneau et Robert, 2003), en ajoutant les critères de tri des documents numériques (Gadoury, 2009-2010). Pour chacun de ces critères, des balises et procédures ont été définies, pour que l'employé réalisant le tri soit apte à évaluer et à trier rapidement les ensembles de photographies.

\subsubsection{Intelligibilité de l'information et qualité du support}

Les principes de base de ce critère sont d'éliminer les photographies qui affichent des défauts techniques liés à la mise au point et à la luminosité, ainsi que d'éliminer les photographies dont le support est en mauvaise condition (Charbonneau et Robert, 2003). Sur le critère des défauts techniques, l'équipe n'a eu aucune hésitation. Pour chacune des séries de photographies, plusieurs poses avaient été réalisées, autant pour ce qui a trait aux ensembles sur négatifs que pour le numérique. II allait de soi que ce critère devait être respecté sans hésitation.

Ce fut un critère déterminant pour faciliter le tri dans les ensembles de photographies telles celle illustrée ci-dessous.
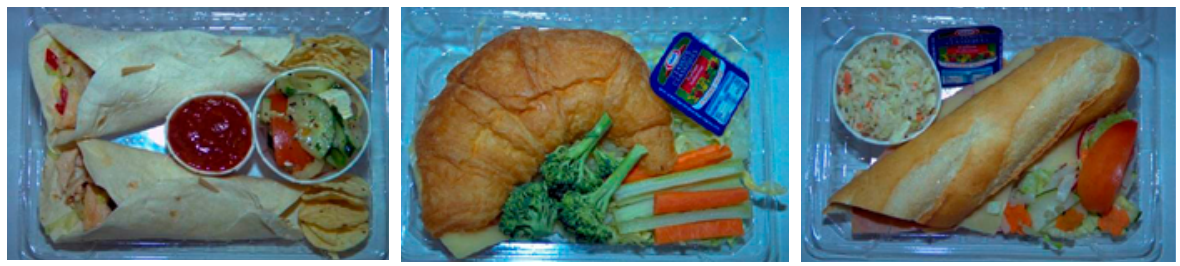

Figures 6-7 et 8: Boîtes à lunch offertes à la cafétéria du CHRDL, vers 2000. 
Cette série comptait plus d'une cinquantaine de clichés d'une dizaine de sandwichs pratiquement toute identique. Seuls la luminosité et des défauts techniques ont permis à l'équipe de faire le tri.

Pour ce qui a trait à la qualité du support, l'équipe a été plus conciliante. S'il y avait une possibilité de conserver la photographie indépendamment de son support, nous l'appliquions, afin de conserver le contenu à défaut de pouvoir conserver le contenant. Une procédure simple a même été rédigée pour récupérer les photographies sur un support désuet (par ex.: la numérisation des épreuves et des négatifs en mauvais état).

Pour les photographies en format numérique, la conversion a été encore plus systématique et la qualité du support encore moins prise en considération. "Lorsque les archives en format électronique arrivent dans les centres d'archives, surtout lors de l'acquisition de fonds privés, les éléments informatiques ont souvent dépassé leur espérance de vie.» (Gadoury, 2009-2010)

La pérennité des supports informatiques (disquettes, CD et DVD), au-delà de leur qualité, est souvent assez courte. Bref, plusieurs disquettes et $C D$ avaient déjà atteint leur espérance de vie lors de leur acquisition par le Service de gestion des documents administratifs. La conversion technique était pour l'équipe une voie intéressante. Pérennité et qualité du support, ainsi que conversion technique, sont d'ailleurs des critères importants dans la sélection des documents électroniques quels qu'ils soient (Ducharme, 2001). De nombreuses photographies numériques ont donc été transférées dans IntelliGID et le support d'origine supprimé.

\subsubsection{Besoins des usagers et mission du centre d'archives}

Appliquer ce critère de tri a été facile pour l'équipe, car les besoins de nos usagers étaient déjà bien connus. Les trois demandes les plus fréquentes étaient l'identification d'événements, des emplacements et des personnes. Il a été très facile d'identifier les photographies à conserver en fonction de ces besoins, même pour la personne de l'externe qui a effectué le tri. Les ensembles pouvant démontrer la pertinence du projet de gestion des documents administratifs (démonstration des résultats aux gestionnaires) ont aussi fait l'objet d'une attention particulière. 
Pour ce qui est de la mission du Service de gestion des documents administratifs, elle est intimement liée à la mission de santé et de services sociaux du CSSS du Nord de Lanaudière. Donc, il a été retenu dans nos principes de tri que les photographies illustrant des soins de santé ou de services sociaux devaient être éliminées avec plus de modération, le but ultime étant la conservation et la mise en valeur du patrimoine québécois dans ce secteur d'activité.

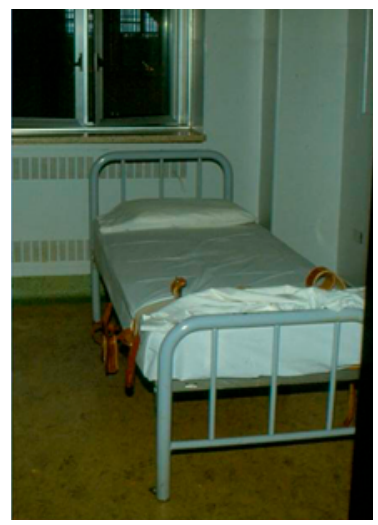

Figure 9: P1 303 - S01 Local du service de psychiatrie courte durée, septembre 1988

La photographie ci-dessus est un bon exemple d'une série de photographies qui a été conservée en fonction de ce critère. Lors de sa construction, I'Hôpital Saint-Charles (aujourd'hui CHRDL) était un hôpital à vocation psychiatrique uniquement. Ces photographies sont donc directement liées à notre mission originale (les soins en santé mentale). D'ailleurs, encore aujourd'hui, la plupart des services en santé mentale sont situés dans le nord de la région.

\subsubsection{Sujet représenté}

Pour faciliter l'application de ce critère de tri, l'équipe a élaboré une liste de sujets à conserver et une liste de sujets à rejeter. C'est d'ailleurs la recommandation que font Normand Charbonneau et Mario Robert dans leur ouvrage (Charbonneau et Robert, 2003). II était alors plus facile pour la personne effectuant le tri de faire une sélection rapide dans les séries et les clichés. 
Dans la section à conserver, il y avait les photographies liées aux: grands événements, conférences de presse, projets de construction, comités et conseils, soins de santé et services sociaux, locaux et bâtiments, réalisations majeures dans les différents services, cérémonies et galas, portraits des directeurs et gestionnaires, activités sociales, dons et activités de la Fondation, formations offertes à l'interne, etc. Dans la section à jeter, il y avait les photographies liées aux: patients, soins de base, plaies, à la chirurgie, dossiers de poursuites judiciaires, etc.

\subsubsection{Intention derrière la photographie}

Le principe de base de ce critère est de sélectionner les photographies en fonction de l'importance que l'on donne aux différentes intentions derrière la création de la photographie: I'intention du photographe, l'intention du sujet photographié, l'intention du client, l'intention du technicien qui fait les retouches et l'intention de la personne qui regroupe les photographies ou les commente (Charbonneau et Robert, 2003).

Pour l'équipe de la gestion des documents administratifs, il était clair que l'intention du photographe était très importante, ainsi que celle du client et du sujet photographié, qui allaient souvent de pair. Une grande partie des photographies de la collection ont été réalisées par le technicien en audiovisuel ou des photographes professionnels expressément engagés par l'organisation pour couvrir un événement. Les autres découlaient de commandes faites par les différents services, dans un but précis. Ces séries reflétaient très bien les besoins et les évolutions de l'organisation, donc l'équipe leur a porté une attention particulière, réduisant le nombre de photographies rejetées.

L'équipe a également retenu plusieurs ensembles qui avaient été regroupés et commentés par une personne de l'organisation, tels les albums. "[L]'archiviste s'interdit de modifier la structure des albums établie par le producteur de fonds»(Charbonneau et Robert, 2003), donc l'équipe a conservé les albums recueillis et n'a pas effectué de tri dans ceux-ci. 


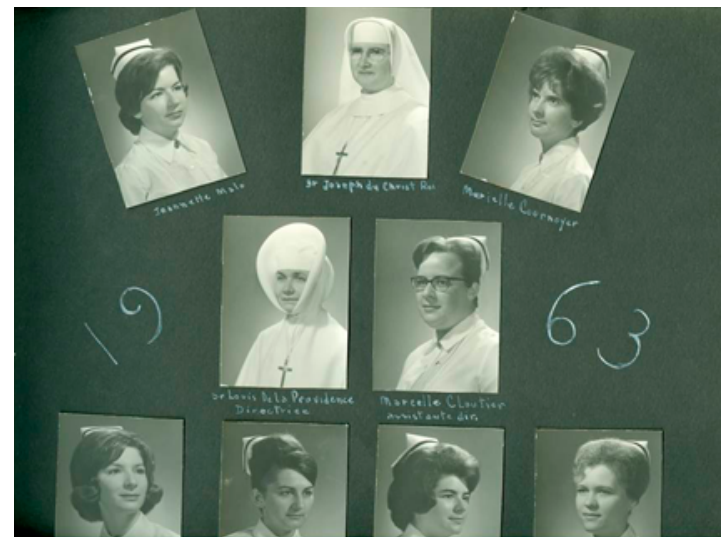

Figure 10: Page de l'album illustrant les enseignantes et finissantes de l'École des infirmières de l'Hôpital Saint-Charles, 1963

L'École des infirmières existait depuis les années 1920 à Saint-CharlesBorromée. Avant d'être postées à l'Hôpital Saint-Charles, elles suivaient leur enseignement à l'Hôpital Saint-Eusèbe, le premier hôpital de la ville. Plusieurs albums de ce genre ont été donnés au service des documents administratifs. La page de l'album reproduite ci-dessus est un bon exemple des albums qui ont été conservés dans la collection sans qu'aucune modification ne soit apportée, ainsi que de l'utilité de ce critère de tri.

Les diverses intentions derrière la photographie y sont bien visibles. La mission du photographe est de réponde à la demande de l'École des infirmières d'immortaliser l'image de leurs finissantes (comme les écoles le font encore aujourd'hui), alors que l'intention du client est de démontrer la réussite de ses élèves et de faire la promotion de l'école. Pour le sujet photographié, c'est le moyen de commémorer cet événement majeur, de se créer un souvenir. Même l'intention de la personne qui a constitué l'album est claire: il s'agit de consigner l'histoire de l'École des infirmières et d'illustrer la réussite de toutes ses étudiantes.

\subsection{5. Éléments d'identification disponibles}

Ce critère de tri n'a pas été déterminant pour la sélection des photographies pour la grande majorité de la collection. Comme l'équipe bénéficiait des éléments d'identification (autant pour la description que pour l'indexation) pour la plupart des séries (inscriptions du technicien 
en audiovisuel sur les enveloppes, documents d'accompagnement, etc.) et comme il était possible d'aller chercher l'information manquante à plusieurs endroits (publications internes, employés de l'organisation, documents historiques, retraités, etc.), très peu de photographies ont été évaluées en fonction de ce critère. Au contraire, l'employé qui faisait le tri devait systématiquement utiliser une des solutions décrites dans la section Description et indexation des photographies, pour aller chercher les éléments manquants.

Les seuls ensembles éliminés par ce critère ont été les photos de chirurgie et les photographies de plaies. En plus de ne représenter que peu d'intérêt pour les utilisateurs, leur description, sans connaissance médicale, était impossible. La plupart du temps, il s'agissait de diapositives dans une boîte, sans datation ni document d'accompagnement. Parfois, certaines photographies en lien avec des projets de construction, telle la photographie d'un trou dans un mur (sûrement prise pour des réclamations aux assurances), ont aussi été éliminées grâce à ce critère.

\subsubsection{Rareté et originalité de l'information}

Le principe de base de ce critère de tri est de sélectionner les photographies selon la rareté du contenu, donc de sélectionner les photographies qui sont originales et qui illustrent un sujet rare (Charbonneau et Robert, 2003). Ce critère a beaucoup été utilisé pour faire le tri des ensembles de photographies numériques pour permettre un élagage plus sévère.

Comme le nombre de poses est souvent énorme, plusieurs photographies font double emploi, c'est-à-dire qu'elles illustrent le même sujet, parfois avec un angle différent, une luminosité différente, une posture différente du sujet photographié, etc. Ces petites variantes ne sont pas nécessairement suffisantes pour faire d'une photographie un objet original et donc de faire partie de la sélection. (Gadoury, 2009-2010)

L'employé chargé de faire le tri avait comme consigne d'éliminer automatiquement les photographies faisant double emploi. 
Le critère de rareté et d'originalité de l'information a aussi permis à l'équipe de justifier la conservation de plusieurs photographies. Par exemple, dans l'ensemble de la collection, il existe peu de photographies illustrant la construction des différents bâtiments occupés par le CSSS du Nord de Lanaudière, et encore moins des équipes ayant réalisé les travaux, donc elles sont vraiment précieuses. Très peu d'élagage a été fait dans ces séries.

Originalité et rareté peuvent aussi faire référence au support de la photographie. S'agit-il de l'épreuve ou du négatif? Sur ce point, l'équipe a été beaucoup plus souple. La plupart du temps, la même valeur était donnée au négatif et à l'épreuve, tout simplement parce qu'il arrivait fréquemment que la série ne contienne que l'un ou l'autre des supports. À défaut d'avoir l'original, la copie était conservée. De plus, pour faciliter la consultation éventuelle des photographies par les utilisateurs, dans la majorité des cas, les épreuves qui accompagnaient les négatifs ont été conservées. Le même tri était effectué et les mêmes poses conservées pour les deux supports.

\subsubsection{Ancienneté}

L'ancienneté des photographies a été un critère de sélection déterminant lors du tri de la collection. Tout d'abord, il était important pour l'équipe de conserver des photographies provenant de tous les âges pour illustrer les différents parcours de santé et services sociaux dans le nord de Lanaudière, mais les photographies anciennes (avant 1970 et avant l'engagement du technicien en audiovisuel) ont été presque toutes conservées. Elles étaient plus rares bien sûr dans la collection, mais aussi dans les autres centres $d^{\prime}$ archives de la région.

Par exemple, une série de photographies réunies dans un vieil album représente l'aménagement et l'intérieur du premier hôpital du nord de la région, l'Hôpital Saint-Eusèbe. À notre connaissance, il s'agit des seules photographies illustrant I'Hôpital Saint-Eusèbe à cette époque. Toutes les photographies ont été conservées sans exception.

\subsubsection{Qualités esthétiques}

Ce critère a été appliqué de façon égale à peu près à toutes les séries. II s'agissait surtout de choisir laquelle des photographies était la plus belle, la plus réussie, celle où tous les gens étaient souriants, par exemple. 
Il a beaucoup servi pour le tri des séries liées à des galas et à des remises de prix. De nombreuses poses illustraient les récipiendaires, les fêtés, les conférenciers, les animateurs. Les plus «jolies» ont été choisies. Le but étant de conserver principalement les photographies qui présentaient les meilleures aptitudes pour la diffusion et les expositions futures.

\subsubsection{Communicabilité}

Le principe de base de ce critère de tri est de sélectionner les photographies en fonction de leur accessibilité, c'est-à-dire si elles sont libres de droits, s'il n'y a pas de restriction de consultation ou d'accès, ou si elles ne font pas obstacle à la confidentialité (Charbonneau et Robert, 2003). Les photographies liées aux patients et aux dossiers médicaux ont justement été éliminées à cause de ce critère. Toutefois, les autres photographies de la collection ont toutes été prises dans le cadre du travail des intervenants et dans le cadre d'événements publics. À priori, aucune d'entre elles n'était visée par une restriction d'accès ou de diffusion.

\subsection{Conservation des photographies}

Pour la conservation de la collection de photographies, l'équipe devait choisir du matériel peu coûteux (ressources limitées), peu volumineux (espaces limités) et aussi très simple pour faciliter le repérage et la consultation. Comme le Service de gestion des documents administratifs était sous la responsabilité de la bibliothèque du CHRDL depuis 2006, l'équipe a pu utiliser une partie du budget du matériel de bureau pour acheter le matériel de conservation nécessaire.

Pour la conservation des négatifs, des épreuves et des diapositives, des pochettes protectrices pour les archives photographiques ont été achetées auprès de fournisseurs d'équipements d'archivage. Toutes les photographies ont été retirées de leur contenant d'origine et insérées dans les pochettes protectrices. Pour l'enveloppe «extérieure», de simples cartables ont été choisis. Pour l'identification, des étiquettes utilisées en bureautique ont été achetées.

Toutes les photographies encadrées ont également été retirées de leur cadre et insérées dans les pochettes protectrices. Les albums ont été rangés dans des boîtes d'archivage adaptées à leur format. Au total, 
après le tri effectué des 11000 photographies (et qui totalisaient une trentaine de boîtes), onze cartables occupent une seule tablette et une dizaine de boîtes d'albums en occupent une autre.

Pour les photographies numériques, l'équipe a décidé de les verser dans IntelliGID, indépendamment de leur support (qui était souvent déjà désuet). Le processus est tout de même assez rapide. Même en renommant les fichiers en fonction de la cote de classification attribuée à chacune des photographies, une quinzaine de minutes suffisent pour insérer une série dans le logiciel.

\subsection{Diffusion des photographies}

Pour la diffusion des photographies, l'équipe s'est surtout concentrée sur la numérisation des photographies. Pour chaque série, entre deux et dix photographies ont été sélectionnées pour être numérisées. Le but était de fournir aux utilisateurs une idée sommaire du contenu de la collection en rendant disponibles quelques exemples dans chacune des séries.

Les photographies numérisées ont été insérées dans le logiciel de la bibliothèque. La plupart des utilisateurs étaient déjà familiers avec cet outil et l'accès n'en était pas restreint comme pour IntelliGID. Lorsque les usagers sont intéressés par une série, ils viennent ensuite la consulter en entier à la bibliothèque, soit dans les cartables si ce sont des négatifs, des diapositives et des épreuves, ou dans IntelliGID si les photographies sont en format numérique.

La diffusion s'est uniquement faite à l'interne pour l'instant. Quelques diffusions à l'externe, comme cette publication dans le journal organisationnel ont été réalisées, mais sans plus.

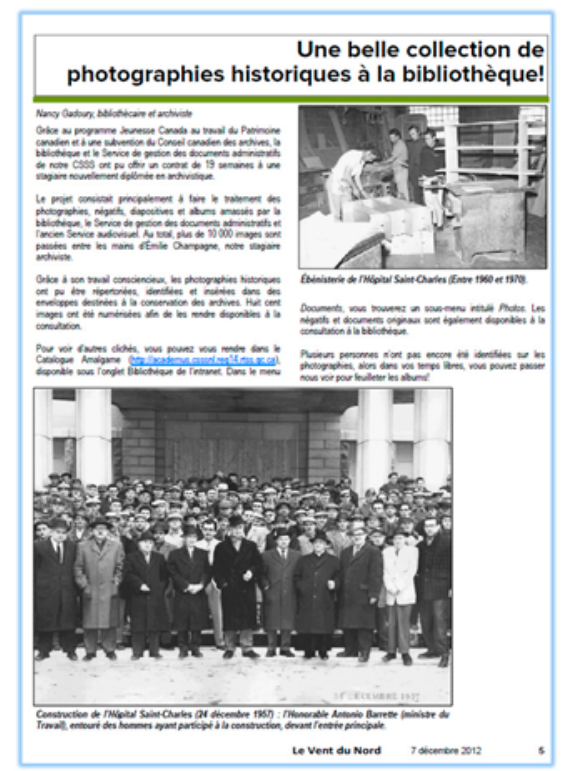

Figure 11: Article publié dans le Vent du Nord, journal organisationnel du CSSS du Nord de Lanaudière 
Les utilisateurs de l'externe sont les bienvenus, mais peu de ressources ont été attribuées à cette partie du projet. Éventuellement, l'équipe envisage de participer à des expositions de photographies anciennes qui sont monnaie courante dans la région.

\section{RÉSULTATS}

Malgré ces résultats enthousiasmants, il a été très difficile de démarrer le projet de traitement de la collection de photographies du CSSS du Nord de Lanaudière. Comme le projet de gestion des documents administratifs n'avait obtenu aucun budget et aucune ressource humaine supplémentaire et qu'il était déjà, à lui seul, une énorme charge de travail pour l'équipe, il a fallu compter sur des ressources externes pour pouvoir le réaliser.

L'équipe a dû convaincre les gestionnaires pour obtenir une autorisation pour faire une demande de subvention dans le cadre du programme Jeunesse Canada au travail de Patrimoine Canada. Dans le cas où la subvention était obtenue, les gestionnaires s'engageaient à fournir les sommes pour participer au projet, dans ce cas-ci, environ $50 \%$ du salaire de la personne engagée. Donc, tout au long de la rédaction du projet de traitement de la collection des photographies, étaient pris en compte les critères de sélection du programme de subvention. L'équipe a opté pour faire une demande pour le stage de fin d'études (minimum de cinq mois d'engagement), plutôt que le programme d'emploi d'été. II était clair que le projet était trop vaste pour être complété en un été.

Par chance, l'équipe a obtenu la subvention et un premier budget a été investi dans le projet. Au bout de cinq mois, après la démonstration de résultats visibles aux gestionnaires, ceux-ci ont accepté de prolonger le contrat de la personne engagée pour trois autres mois. L'équipe a pu ainsi terminer le traitement de la collection en entier et avancer dans le projet de diffusion.

Présentement, l'équipe a dû mettre la suite du projet en veilleuse à cause de la nouvelle fusion des services de santé et de services sociaux survenue au $1^{\text {er }}$ avril 2015 avec la Loi 10. II en va de même pour tout le projet de gestion des documents administratifs (étapes 11, 12 et 13). Avec cette nouvelle fusion, six établissements de santé et services 
sociaux ont été fusionnés (CSSS du Nord de Lanaudière, CSSS du Sud de Lanaudière, Agence de santé et de services sociaux de Lanaudière, Centres jeunesse de Lanaudière, Centre de réadaptation la Myriade et le Centre de réadaptation physique le Bouclier) créant le Centre intégré de santé et de services sociaux de Lanaudière.

Comme en 2003-2004, il faut réviser tous les processus de gestion des documents administratifs, fusionner les différents systèmes de gestion documentaire et faire des choix pour l'avenir. Plusieurs autres beaux projets arrivent à l'horizon, mais il faudra encore un peu de temps pour en voir le résultat.

\section{Nancy Gadoury}

\section{BIBLIOGRAPHIE}

ASSOCIATION QUÉBÉCOISE D'ÉTABLISSEMENTS DE SANTÉ ET DE SERVICES SOCIAUX (AQESSS). (2009). Recueil de règles de conservation des documents des établissements de santé et services sociaux du Québec. Montréal, Québec: Association québécoise d'établissements de santé et de services sociaux.

ARCHIVES NATIONALES DU QUÉBEC. (2000). Guide d'aménagement d'un centre d'archives. Sainte-Foy, Québec: Publications du Québec.

CASAULT, D. et CHARBONNEAU, N. (2001). Plan des mesures d'urgence pour les documents d'archives. Québec, Québec: Publications du Québec.

CHARBONNEAU, N. et ROBERT M. (2003). La gestion des archives photographiques. Sainte-Foy, Québec: Presses de I'Université du Québec.

DUCHARME, D. (2001). L'identification de critères d'évaluation pour les archives informatiques: Enquête auprès des archivistes québécois, Archives 32(2), 17-32.

GADOURY, N. (2009-2010). L'évaluation des photographies en format numérique, Archives 41(1), 31-43. 
SCHWARTZ, J.M. (2002). Coming to terms with photographs: descriptive standards, linguistic « othering " and the margins of archivy, Archivaria 54, 142-171.

SMITH, F. (2008-2009). La collection, unité archivistique à définir: l'exemple des archives photographiques, Archives 10(1), 15-26. 